\section{MARS: a RISC-based architecture for Lisp}

\author{
Hung-Chang Lee, Feipei Lai, \\ Jenn-Yuan Tsai and Tai-Ming Parng \\ Department of Electrical Engineering and \\ Department of Computer Science, National \\ Taiwan University, Taipei, Taiwan, R.O.C.
}

\begin{abstract}
A RISC-based chip set architecture for Lisp is presented in this paper. This architecture contains an instruction fetch unit (IFU) and three processing units-integer processing unit (IPU), floating-point processing unit (FPU), and list processing unit (LPU). The IFU feeds instructions to the processing units and supports fast procedure call/return and branch, the IPU and FPU execute operations of different data type, and the LPU handles the Lisp runtime environment, dynamic type checking, and fast list access. In this architecture, the critical path of complex register file access and ALU operation is distributed into the LPU and IPU, and the tracing of a list can be done quickly by the non-delayed car or $c d r$ instructions of the LPU. Performance simulation shows that this architecture would be about 6.2 times faster than SPUR and about 2.2 times faster than MIPS-X.
\end{abstract}

\section{INTRODUCTION}

Lisp, due to its extensibility and flexibility, has gained popularity these days. Nevertheless, the Lisp programming language has some features that are difficult to implement efficiently on conventional computers. These features include frequent function calls, slow list traversal, polymorphic operations, and automatic garbage cell recovery ${ }^{1-3}$.

The Lisp machines, according to Pleszkun's ${ }^{1}$ classification, can be divided into three classes. First, the unspecialized stack-based microcoded Lisp processors (e.g. Symbolics $3600^{2}$, Lambda ${ }^{4}$ ). Second, multiprocessor architectures where each processor serves a specialized function (e.g. Fairchild FAIM-1 ${ }^{5}$ ). Third, multiprocessor systems composed of pools of identical processing elements aiming for high performance through concurrent evaluation of different parts of a Lisp program on separate processors (e.g. EM-3 ${ }^{6}$ ). Another class of Lisp machine designed recently is a RISC-like architecture with some enhancements to support Lisp, such as SPUR ${ }^{3}$, or by appealing to a compiler to reduce the hardware complexities such as MIPS-X ${ }^{7-9}$.

A limited instruction set suitable for Lisp execution is presented, and a RISC-based architecture, based on this instruction set, is designed. In fact, the architecture model of MARS has three layers. The first layer is the Lisp environment administrator and list travelling access. The second one is the general computational unit, and the third is the instruction feed and control transfer unit. Each layer has its respective chip to carry out its task. These synchronized chips take advantage of instruction format parallelism and achieve parallel execution whenever possible.

The next section gives an overview of the systems. This is followed by a description of the microarchitecture and instruction pipeline of MARS. The next section gives a performance evaluation using the simulation tools. Conclusions and status are stated in the last sections.

\section{SYSTEM OVERVIEW}

MARS $^{10}$ is a VLSI processor board for Lisp processing. Inside each board, shown in Figure 1, there are CPU chips, i.e., IFU (Instruction Fetch Unit) and IPU (Integer Processing Unit) as well as special chips, FPU (Floating-point Processing Unit) and LPU (List Processing Unit). Each processor board has separate Instruction, Address, and Data buses.

IFU, built on a single chip together with a $4 \mathrm{kB}$ instruction cache, is the buffering, controlling mechanism between the instruction cache and the datapath chips (IPU, FPU, LPU). It is designed to

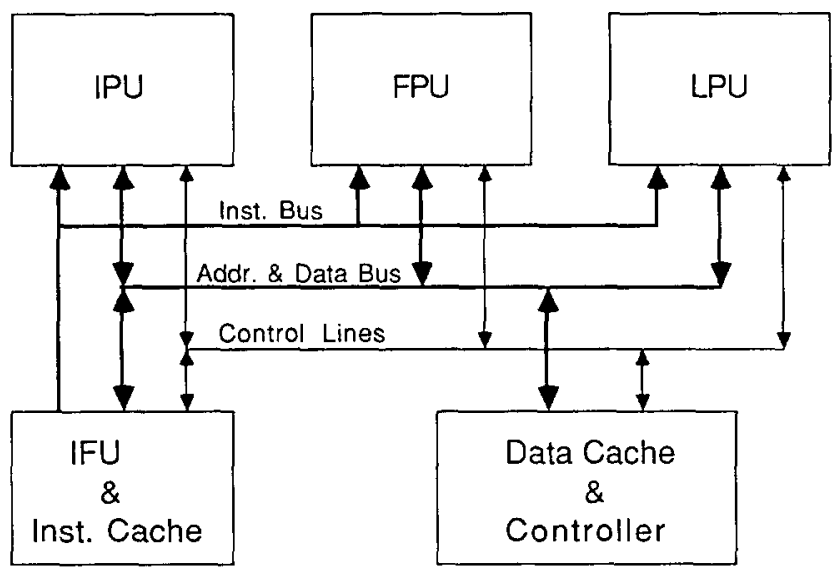

Figure 1 Single board block diagram 
MARS: a RISC-based architecture for Lisp: Hung-Chang Lee et al.

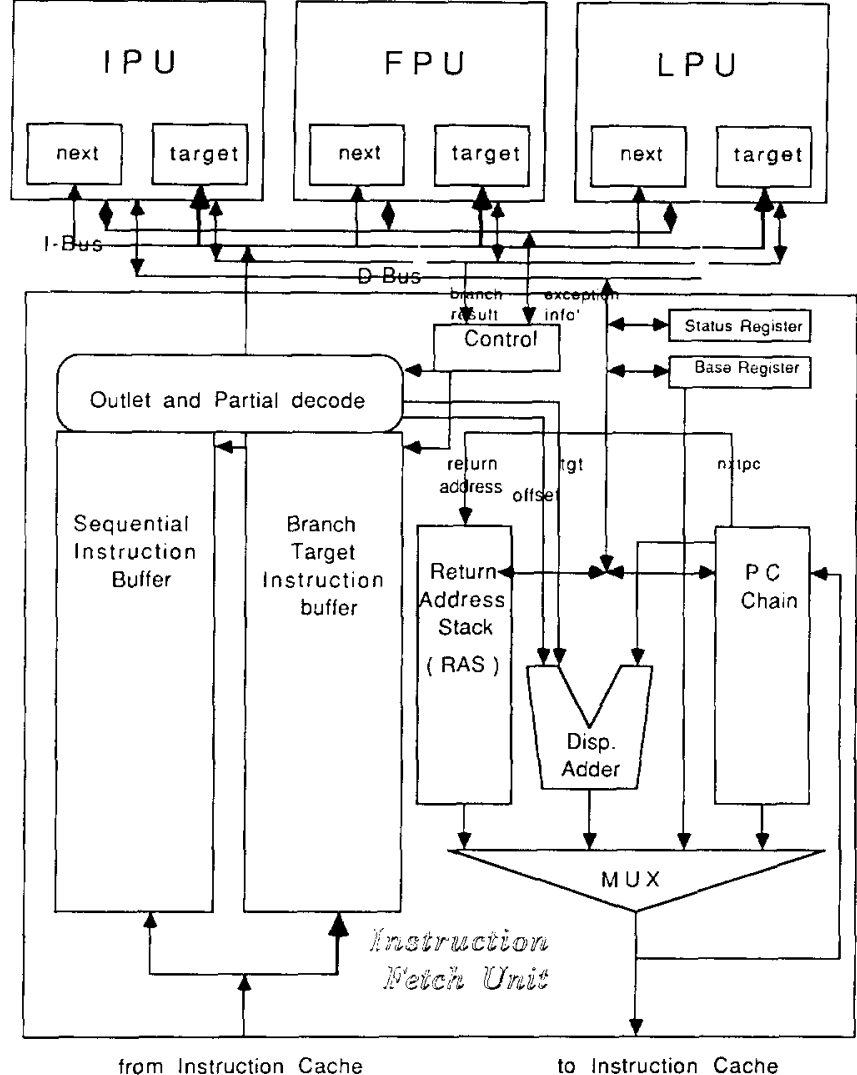

Figure 2 The IFU block diagram

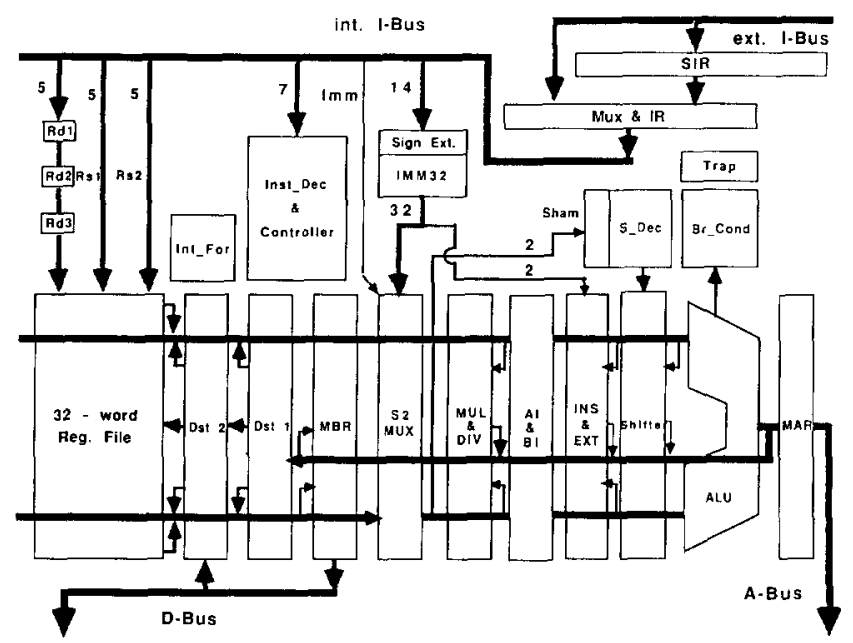

Figure 3 The IPU block diagram

interleave instruction fetch and execution and achieve coordinated execution among IPU, FPU, and LPU. The block diagram is given in Figure 2, in which there are a remote PC (Program Counter) chain, a displacement adder, a return address stack (RAS) to store PC values for call/return instruction pairs, and dual instruction buffers for holding sequential and branch instruction streams.
The IPU, shown in Figure 3, retains the integer datapath and some control part of a common RISC $\mathrm{CPU}^{11}$, performing integer arithmetic, shifts, logical operations, and address calculation for data operands of all datapath chips. There are a flat 32-word register file, a 2-level internal forwarding latch, and a shifter.

The LPU, shown in Figure 4, provides hardware primitives for list processing, such as car, cdr, cons, rplaca (replacea) and rplacd (replaced). It features a big windowed register file to expedite procedure calls, a tag manipulation datapath, and a control register for shallow binding.

The CCMMU (Cache Controller and Memory Management Unit) is responsible for the operation of local data cache on each processor board, addressing translation, and data coherency protocol among processors. Local data cache will be as large as $128 \mathrm{kB}$ and data will be heuristically prefetched in the face of a pointer or list. Lock-up free cache design together with the cache coherency protocolphoenix-is proposed in Reference 12.

\section{Why separate IFU, IPU, and LPU?}

At first, we intended to adopt an integrated LPU architecture like Symbolics 3600 , but owing to the unique configuration and some other considerations

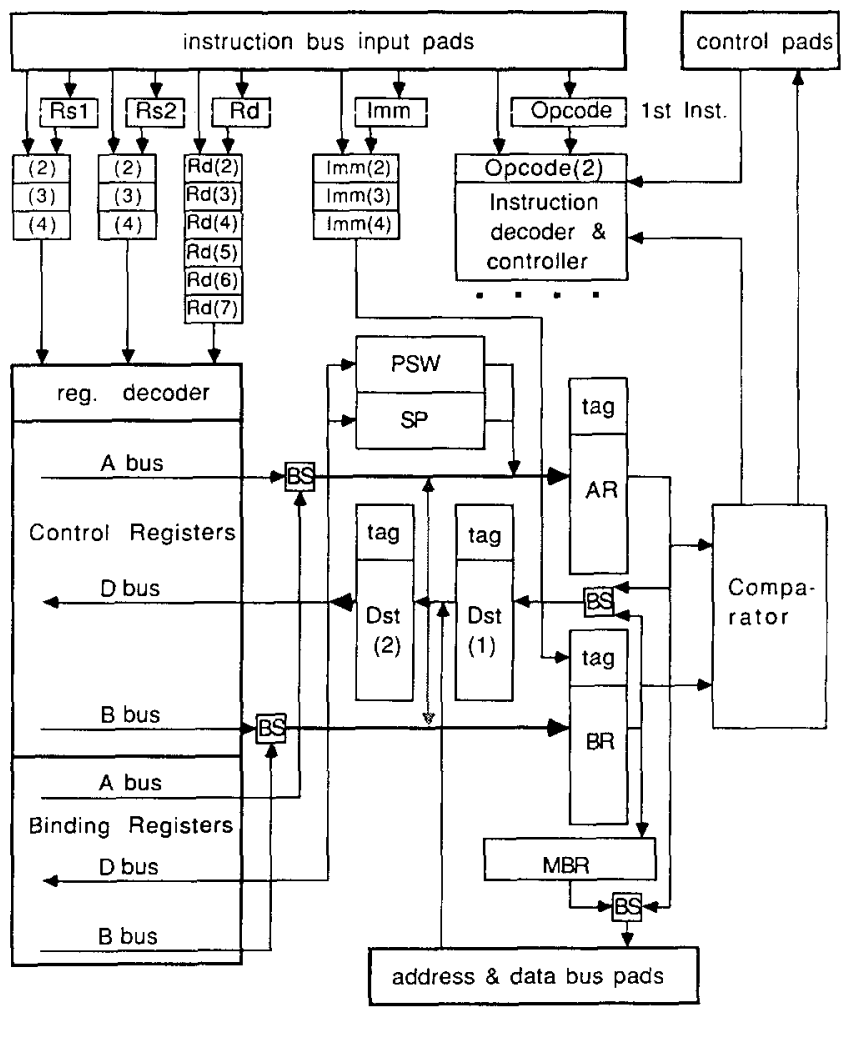

Figure 4 The LPU block diagram 
inside MARS (MARS can run conventional languages, for instance $C$, in different ways $)^{10,13}$, an on-chip instruction cache inside the LPU will not provide any speed advantage when all the chips wait for instructions coming from the instruction cache and then decode their own instructions. Therefore, we decided to separate the instruction fetch unit for conventional LPU, and build an IFU to accommodate the remote $\mathrm{PC}$ unit and the necessary logic for buffering and control of instruction access. This decision did cause some problems, thereby influencing our designs for the instruction set and microarchitecture, but it provided us with valuable experience in dealing with such an arrangement, and the result can still meet our initial requirement.

\section{MICRO-ARCHITECTURE}

\section{Instruction set}

The instruction set of the LPU ${ }^{13}$, shown in Table 1 , was carefully designed to speed up the execution of Lisp programs and to reduce the time wasted by traffic between the LPU and the IPU/FPU. In order to reach this goal, there are some instructions executed parallel by the LPU, the IPU and/or the FPU. Parallel execution of the IPU and LPU happens when the IPU executes some ALU operation and the LPU checks the corresponding tag of source registers and generates exceptions if the data types of operands are neither fixed numbers nor characters. Another parallel execution happens when the LPU loads or moves data, used by the IPU or FPU, into a frame register. Besides the IPU and FPU, the LPU also writes this data into the corresponding register. With this parallel execution, we need not take extra instructions to transfer data from the LPU to the IPU or to the FPU. The instructions of the LPU with this kind of parallel execution are car, pop, load and mov.

\section{List primitives}

The hardware-implemented list primitives are car,

Table 1 LPU instruction set

Exception

\begin{tabular}{|c|c|c|c|}
\hline $\begin{array}{l}\text { List primitives } \\
\quad \text { car_b } \\
\text { car_c } \\
\text { cdr } \\
\text { cons } \\
\text { rplaca } \\
\text { rplacd }\end{array}$ & $\begin{array}{l}\text { BRd,Rs1 } \\
\text { CRd,Rs1 } \\
\text { Rd,Rs1 } \\
\text { Rd,Rd1,Rs2 } \\
\text { Rs1,Rs2 } \\
\text { Rs1,Rs2 }\end{array}$ & $\begin{array}{l}\text { BRd =M[Rs1_data \& } \sim 07] \\
C R d(i p u, f p u, I p u)=M[R s 1 \text { data \& } \sim 07] \\
R d=M[R s 1 \text { data }\langle 31: 4\rangle \mid 04] \\
M[R s 1 \text { data \& } \sim 07]=R s 2, R d=R s 1 \\
M[R s 1 \text { data \& } \sim 07]=R s 2 \\
M[R s 1 \text { data }\langle 31: 4\rangle \mid 04]=R s 2\end{array}$ & $\begin{array}{l}E \\
E \\
E \\
D \\
D \\
D\end{array}$ \\
\hline $\begin{array}{l}\text { Stack } \\
\text { push } \\
\text { pop_b } \\
\text { pop_c }\end{array}$ & $\begin{array}{l}\text { Rs2 } \\
\text { BRb } \\
\text { CRd }\end{array}$ & $\begin{array}{l}S P=S P+1, M[S P]=R s 2 \\
B R d=M[S P], S P=S P-1 \\
C R d(i p u, f p u, I p u)=M[S P], S P=S P+1\end{array}$ & \\
\hline $\begin{array}{l}\text { Data transfer } \\
\text { mov_b } \\
\text { mov_c } \\
\text { I_load_b } \\
\text { I_load_c } \\
\text { I_store } \\
\text { f_to_I } \\
\text { I_load_f } \\
\text { I_store_f }\end{array}$ & $\begin{array}{l}\text { BRd,Rs2 } \\
\text { CRd, Rs2 } \\
\text { BRd,Rs1 } \\
\text { CRd,Rs1 } \\
\text { Rs1,Rs2 } \\
\text { Rd,Rs1 } \\
\text { Rd,Rs1 } \\
\text { Rs1,Rs2 }\end{array}$ & 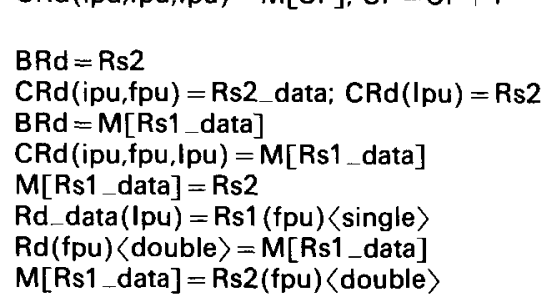 & \\
\hline $\begin{array}{l}\text { Tag } \\
\text { load_tag } \\
\text { mov_tag }\end{array}$ & $\begin{array}{l}\text { Rd,Rs1,imm_tag } \\
\text { Rd,Rs1,Rs2 }\end{array}$ & 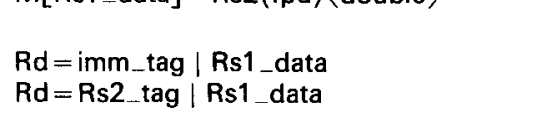 & \\
\hline $\begin{array}{l}\text { Compare branc } \\
\text { I_dcb_rr } \\
\text { I_dcb_ri } \\
\text { I_scb_rr } \\
\text { I_scb_ri }\end{array}$ & $\begin{array}{l}\text { Rs1, Rs2, target } \\
\text { Rs1,imm_tag,target } \\
\text { Rs1,Rs2,target } \\
\text { Rs1,imm_tag,target }\end{array}$ & $\begin{array}{l}\text { cond }=(R s 1 \text { op Rs } 2) \\
\text { cond }=(R s 1 \text { op Imm_tag }) \\
\text { cond }=(R s 1 \text { op Rs } 2) \\
\text { cond }=(R s 1 \text { op Imm_tag })\end{array}$ & \\
\hline $\begin{array}{c}\text { Call/return } \\
\text { cal_jmp } \\
\text { ret_jmp }\end{array}$ & $\begin{array}{l}\text { addr } \\
\text { addr }\end{array}$ & $\begin{array}{l}c w p=c w p+1 \\
c w p=c w p-1\end{array}$ & $\begin{array}{l}A \\
B\end{array}$ \\
\hline $\begin{array}{l}\text { Special } \\
\text { rd_sp } \\
\text { wr_sp } \\
\text { rd_Ipsw } \\
\text { wr_Ipsw } \\
\text { Ipu_wake } \\
\text { Ipu_sleep }\end{array}$ & $\begin{array}{l}\text { Rd } \\
\text { Rs1 } \\
\text { Rd,Lpsn } \\
\text { Rs1,Lpsn }\end{array}$ & $\begin{array}{l}\text { Rd_data }=\text { SP } \\
\text { SP }=\text { Rs1_data } \\
\text { Rd = LPSW }(\text { Lpsn }) \\
\text { LPSW }(\text { Lpsn })=\text { Rs1 } \\
\text { Ipu wake up } \\
\text { Ipu sleep }\end{array}$ & \\
\hline
\end{tabular}




\section{MARS: a RISC-based architecture for Lisp: Hung-Chang Lee et al.}

$c d r$, cons, rplaca and rplacd. The car and $c d r$ instructions are similar to a "load" instruction except that the least two significant bits of the address are masked with 00 or 01 , respectively. In the LPU pipeline stage, there is no delay for a load instruction, so the trace of a list could be done fast by the $c d r$ and car instructions. The rplaca and rplacd instructions are similar to the "store" instruction, except for masking the address bits as in $c a r$ and $c d r$. The cons instruction executes the action of rplaca by using the global register R7 (free cons cell pointer) as the address and moves R7 to the destined register. A complete cons primitive in Lisp could be done by a cons instruction following a rplacd instruction to replace the cdr of this cons cell, and a car instruction to update the free cons cell pointer. All the above instructions are executed with parallel type-checking. If the data type of the source address register is not cons or nil, it will result in an exception.

\section{Stack operation}

The stack operation can be done in one instruction cycle by the push and pop instructions of the LPU. The stack pointer register (SP) in the LPU decreases by 2 (distance of double-word) before executing the push instruction and increases by 2 after executing the pop instruction automatically. The push and pop instructions are mainly used when binding or unbinding the special variables and when saving or restoring frame windows. The pop instruction, as car and $c d r$, is a non-delay load instruction which can supply data for the next LPU instruction without delay. The contents of the stack pointer register can be read or written by $r d_{-} s p$ or $w r_{-} s p$ instructions.

\section{Data and tag transfer}

The data transfer instructions include: the load instruction which loads data from memory, the mov instruction which moves data from a register to another register, the load_f and store $f$ instructions which generate the address for the FPU, and the $f_{-} t o_{-} l$ instruction which transfers data from the FPU to the LPU. Note that the load and mov instructions are executed in parallel by the IPU, FPU and LPU when the destination is a frame register and the action of transferring data from the LPU to the IPU or to the FPU can be done by the mov instruction.

The tag value of a register can be loaded with the immediate tag value packed in an instruction or moved from another register. The data field of the destined register could be the destined register itself or another register which is the second source register specified in the instruction.

\section{Compare \& branch}

In the MARS system, comparison of two operands and branching are executed in one instruction cycle with zero or one delay. The compare \& branch instruction of the LPU compares two operands with eight kinds of conditions encoded in 3-bit condition code and sends the compared result to the other processing units.

\section{Function call and return}

The actions of jumping to a target address and saving of the program counter of function call and return instructions are done by the IFU, while the LPU updates the pointer of the current frame window and checks whether the control register file is being overflowed, underflowed or neither. If an overflow or underflow happens, the LPU causes an exception.

\section{Special instructions}

There are some special instructions of the LPU which could be executed only in kernel mode. The $r d \_l p s w$ and $w r_{-} l p s w$ instructions transfer data between registers and the LPU processing status word which contains the current window pointer, the saved window number and some system status. The lpu_wake and lpu_sleep instructions set the LPU to be active or inactive. When the LPU is inactive, the MARS system is acting as a general-purpose computer without hardware support for Lisp.

\section{Data type and storage}

MARS represents a List pointer or immediate data with a 38-bit tagged word consisting of a 6-bit tag and a 32-bit pointer or data. The data types represented by the tag are shown in Table 2 .

There are three immediate data types: character, fixed number and short floating-point number. The first two are used by the IPU and the latter by the FPU. We decided to represent short floating-point numbers by an immediate type because all the IPU, FPU and LPU have a user-view of a 32-word control register file. The register files are monitored by the LPU as a frame window and saved in the LPU's frame window while executing a function call. The 32-bit short floating-point number has the same width as the data field of the LPU's register; 
Table 2 Data types and corresponding tag values

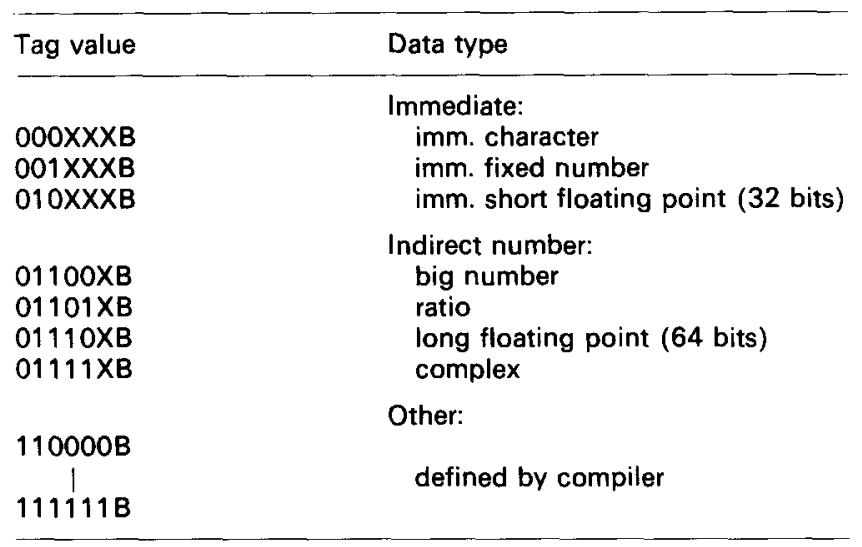

therefore, it can be used as an immediate data and stored in the LPU's frame window.

The type of data (which may be an immediate number, an indirect number, or a list) is recognized by a type-checking hardware in parallel with the execution of data itself - usually under the assumption of integer type, or with the comparison of the LPU-compare-branch instruction. The other 16 tag values are defined by the compiler to identify pointed objects such as string, vector and function.

MARS stores the 38-bit tagged word in memory by two 32-bit words aligned in a double-word boundary (data in the even word and tag in the odd word) with 26 bits unused. The data bus connecting four processing units and cache memory is 64-bit wide -32 bits are needed by the IFU and IPU, 64 bits by the FPU and 38 bits by the LPU. This means that we can load/store one or two 32-bit words in one memory cycle.

A list cons cell consisting of the car's tagged word and the cdr's tagged word is represented by four contiguous 32-bit words aligned in the quad-word boundary of memory. The first half of the double-word contains the car's tagged word and the second one contains the cdr's tagged word. We can access the car's tagged word of a cons cell by setting the least four significant bits of the cell's address to 0000 , and access the cdr's tagged word by setting these four bits to 1000 .

\section{Register structure}

The register structure in the LPU plays the role of a runtime environment administrator. In Lisp, the arguments, local variables and special variables are accessed frequently. These variables are allocated in the register file and maintained in a fast scheme described later. There are two kinds of register files in the LPU; one is the control register file and the other is the binding register file. The control register file, organized as an overlapping frame window structure, is used to keep the activation records of callers and callees. The 32-word register files of the IPU and LPU are mapped to one of the frame windows, so the user can view the control register as a 32-word register frame window whose data may be either integer (in IPU), floating-point number (in FPU), or pointer (in LPU). The binding register file is used to keep the binding value of special variables. We use a shallow binding scheme to bind and restore the special variables.

\section{Control register file}

The control register file is organized as multiple, overlapping, fixed-size frame windows (shown in Figure 5), similar to the multi-window register file of RISC I\&II ${ }^{11}$. However, the control register file differs from that of RISC II in that it has to monitor the 32-word register files of IPU/FPU and keep their contents in the corresponding frame windows in function calling. We designed a mechanism, which will be described later, to map the registers of the IPU/FPU into the control register file of the LPU across function call/return. There are eight frame windows and a total of 136 registers in the control register file, but only 32 registers (one frame window) can be seen at a time by the user. The user's view of the 32-word registers is further partitioned into four eight-word register subframes -i.e., global, input, output, and local. The partition of the 32-word registers is different from that of RISC II, which has 10 registers for global, six for input, six for output and 10 for local, for the reason that Lisp uses more arguments and fewer local variables than $\mathrm{C}$ or Pascal, and this arrangement makes the mapping of the IPU/FPU's registers to the control register file easier. The global frame shared by all windows is used to hold some environment variables such as return value and pointer to the top of heap memory. The input frame is intended to place input arguments from the parent function (caller). On the other hand, the output frame is used to hold and send arguments to the child function (callee). The output frame of the caller is overlapped with the input frame of the callee. Once a function is called, the window viewed by the user switches from caller to callee and the output frame of the caller now becomes the input frame of the callee. The local register frame which does not overlap with the other window is used to store local lexical variables or temporary values. 
MARS: a RISC-based architecture for Lisp: Hung-Chang Lee et al.

\section{IPU/FPU}

LPU

\begin{tabular}{|c|c|c|c|}
\hline A & Ro - R7 & \multicolumn{2}{|c|}{ GLOBAL } \\
\hline$B$ & $R 8-R 15$ & WO.IN & W7.OUT \\
\hline C & R16-R23 & WO.LOCAL & \\
\hline$D$ & R24-R31 & WO.OUT & W1.IN \\
\hline$C$ & $\mathrm{R} 16-\mathrm{R} 23$ & & W1.LOCAL \\
\hline$B$ & $\mathrm{R} 8-\mathrm{R} 15$ & W2.IN & W1.OUT \\
\hline$C$ & $\mathrm{R} 16-\mathrm{R} 23$ & W2.LOCAL & \\
\hline$D$ & R24-R31 & W2.OUT & W3.IN \\
\hline$C$ & R16-R23 & & W3.LOCAL \\
\hline$B$ & $\mathrm{R} 8-\mathrm{R} 15$ & W4.IN & W3.OUT \\
\hline$C$ & $\mathrm{R} 16-\mathrm{R} 23$ & W4.LOCAL & \\
\hline$D$ & R24-R31 & W4.OUT & W5.IN \\
\hline$C$ & $\mathrm{R} 16-\mathrm{R} 23$ & & W5.LOCAL \\
\hline$B$ & $\mathrm{R} 8-\mathrm{R} 15$ & W6.IN & W5.OUT \\
\hline$C$ & $\mathrm{R} 16-\mathrm{R} 23$ & W6.LOCAL & \\
\hline$D$ & R24-R31 & W6.OUT & W7.IN \\
\hline$C$ & R16-R23 & & W7.LOCAL \\
\hline
\end{tabular}

Figure 5 The frame-window structure of the control register file and mapping of corresponding register groups in the IPU/FPU

In the MARS system, only the LPU has a frame-window register structure and there are just 32 registers in the IPU and FPU. How do we retain the IPU's and FPU's register data when executing a function call? We solve this problem by the following mechanisms: First, the 32-word register files of the IPU/FPU are partitioned into four eight-word register groups which are mapped into the four sub-frames of the LPU's current frame window. Figure 5 shows the mapping of the IPU/FPU's 32-word register into the LPU's frame window. $A$ group registers (R0-R7) and $C$ group registers (R16-R23) of the IPU/FPU are always mapped into the global frame and local frame of the LPU's current frame window. In contrast, $B$ group registers ( $\mathbf{R} 8-\mathbf{R} 15)$ and $D$ group registers (R24-R31) of the IPU/FPU are mapped into the input frame and output frame or vice versa, according to whether the current window number of LPU is even or odd. Assuming that the window number of the current function is 0 , then $B$ group registers are mapped into the input frame and $D$ group registers are mapped into the output frame. After calling a child function, the frame window number is increased by one and now the $D$ group registers of the IPU/FPU are mapped into the input frame of window 1, which is the output frame of window 0 (see Figure 6). This means that we do not have to save the eight registers corresponding to the output frame of the current window when executing a function call. Likewise, $A$ group registers mapped into the common global frame of all frame windows do not have to be saved, so only the remaining two eight-word register groups mapped into the local frame and input frame need to be saved into or restored from the corresponding frames of the LPU's current window when executing a function call or return.

The translation from the IPU/FPU's register number to the sub-frames of the LPU's current frame window can be implemented easily by the circuit shown in Figure 7 . The turn signal sent from the LPU is reset to 0 when the number of the LPU's current frame window is even and is set to 1 when

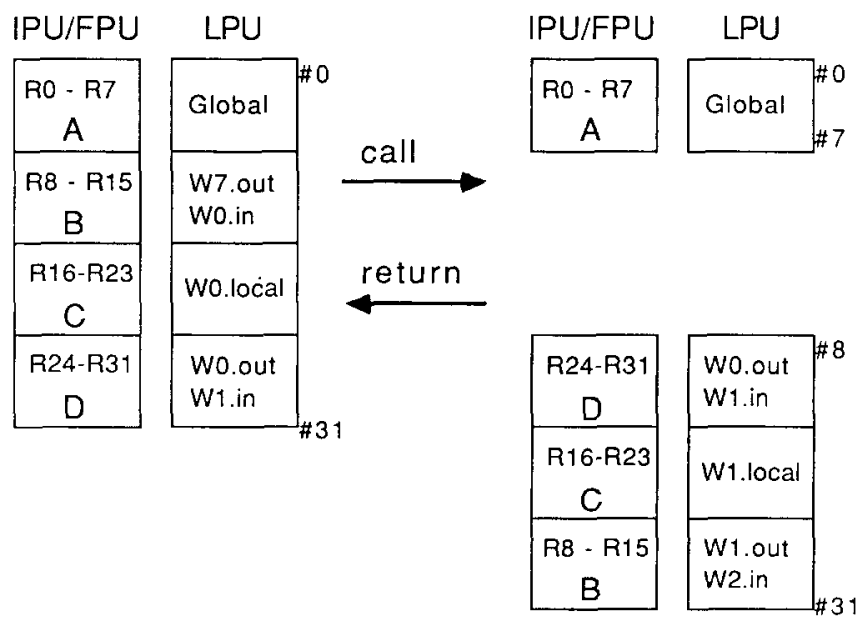

Figure 6 Mapping of the frame window before and after executing a function call and return 


$\begin{array}{lccccc} & R(4) & R(3)=>R(4) & R(3) & \\ & 0 & 0 & 0 & 0 & A \\ \text { TURN = } 0 & 0 & 1 & 0 & 1 & B \\ & 1 & 0 & 1 & 0 & C \\ & 1 & 1 & 1 & 1 & D \\ & 0 & 0 & 0 & 0 & A \\ \text { TURN = 1 } & 0 & 1 & 1 & 1 & D \\ & 1 & 0 & 1 & 0 & C \\ & 1 & 1 & 0 & 1 & B\end{array}$

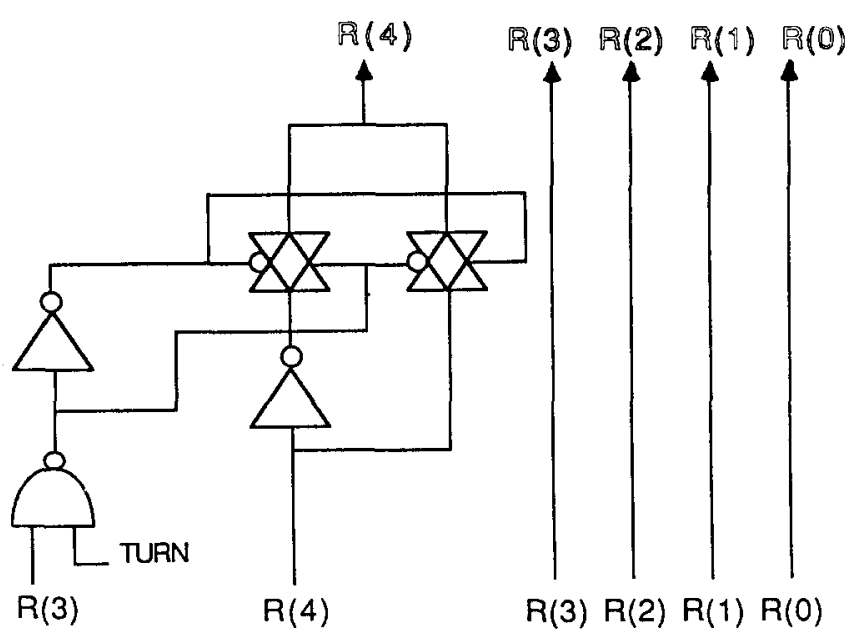

Figure 7 Translation of the IPU/FPU register number

the frame window number is odd. When turn is 0 the translation is an identical one; when turn is 1 the translation maps the register number of $B$ group into the output frame and maps the register number of $D$ group into the input frame.

Apart from the above mapping scheme to reduce the overhead of saving and restoring the IPU/FPU register data, we save them into the LPU's current frame window, parallel with the execution of IPU's instruction. The LPU monitors all the instructions executed by the IPU. When the IPU executes an operation and writes the result back to the register file, it also puts this result on the data bus at the memory cycle. Meantime, the LPU receives the data and writes it back into the corresponding register of the current frame window. With this mechanism, we need not save any IPU register data into the LPU while executing a function call, we only have to restore the necessary IPU register data from the LPU which would be used before the execution of the next function call or before the end of the current function when the called function returns. This overhead would be about two or three instruction cycles per function call, on average.
By using the above mechanisms, the register data of the IPU could be kept in the LPU's control register file with little overhead while executing a function call or return. The multiple, overlapping frame window structure of the control register file in the LPU updates runtime environment very fast. Because the LPU does not execute ALU operations, it can spend more time in accessing the complex register file. On the other hand, the IPU which must spend time in executing ALU operations, has a simple 32-word register file and can access the register more quickly.

\section{Binding register file}

The 32-word binding register file, which has no counterpart register file in the IPU/FPU, is used to store special variables in Lisp. Each special variable corresponds to one register allocated at loading time. We use a shallow binding scheme to handle the binding registers. When a special variable is bound to a new value, the old value in the corresponding register has to be pushed into the binding stack, but when this special variable is unbound, the old value is popped from the building stack and restored to the corresponding register. An example of binding and unbinding of special variables is shown in Figure 8 . The binding registers,

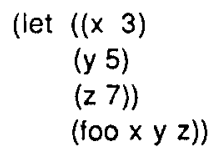

Before let binding \& after (foo $x y z$ )

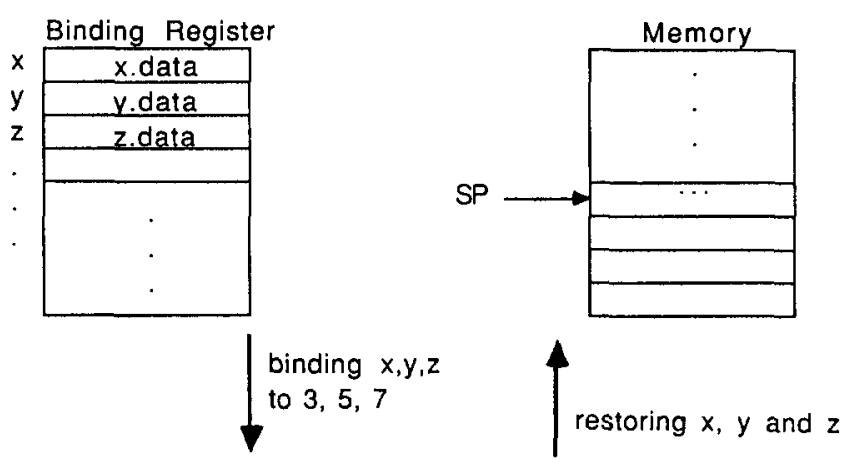

After let binding:
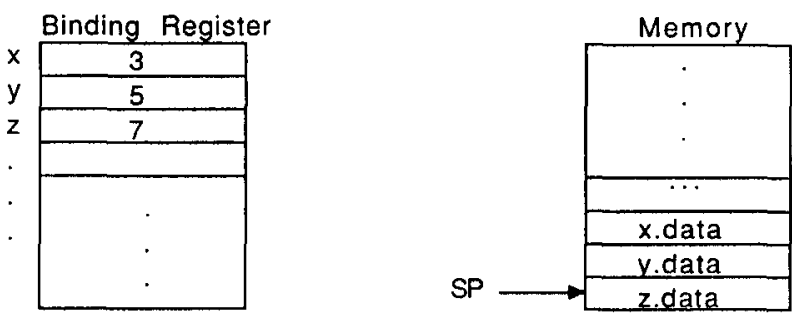

Figure 8 Binding and unbinding of special variables 
which do not have a corresponding register file in the IPU/FPU, can only be used by the LPU's instructions. If they are to be executed by IPU or LPU instructions, they should be moved to the global frame registers. By using the binding registers, we can speed up the access to special variables.

\section{PIPELINE SCHEME AND CONTROL TRANSFER}

MARS uses a front-end instruction fetch stage plus the following two independent four-stage pipelines, shown in Figure 9, attempting to issue and complete an instruction every cycle. The instruction fetch stage issued by the IFU fetches the following instruction after a non-compare instruction and two instructions (plus control transfer target address) after a control transfer instruction. The two four-stage stages are independent but synchronously executed by the IFU and LPU respectively to meet the execution requirements that these two chips demand. For example, one duty of the IPU is to determine the branch condition, so that the register value which determines the condition should be fetched as early as possible. On the other hand, the LPU does not take this responsibility, so the register fetch stage is delayed as far as possible to await the result of external data reference.

Through this kind of pipelined stage arrangement, MARS can support almost-zero-delay branch, super-zero jump, and a non-delayed list access.

\section{Almost-zero-delay branch and super-zero-delay jump}

Branch instructions have a considerable effect on
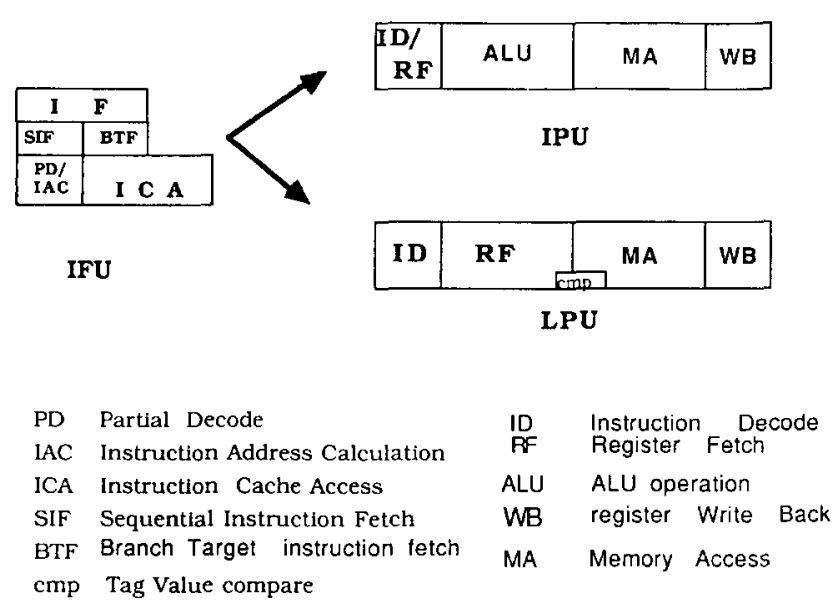

Figure 9 Three kinds of pipeline stage in MARS the performance of pipelined machines ${ }^{14-16}$. Conventional architectures employ additional hardware to deal with this problem. They detect the presence of a branch instruction and put off prefetching until the branch condition has been evaluated, or use some branch-prediction techniques to reduce the number of control flow breaks ${ }^{17,18}$. Recently, a Branch Folding was proposed in the design of CRISP $^{19}$, which folded a non-branch and the following branch instruction to get zero-delay branching. A well-designed IFU adopts the combination of delayed branch, multiple prefetch and early resolution methods to reduce the branch penalty. IFU executes the first two stages of our total six-stage pipeline and issues instructions to the datapath chips (IPU, FPU, and LPU). Some intelligence exists in the IFU when issuing the instruction flow. The partial decode unit of the IFU, executing at the PD (partial decode) stage, can discover the existence of an incoming jump instruction, calculate the address and access the instruction ahead of time. The IFU can absorb that jump instruction and send out the jump target address simultaneously. This mechanism makes a super-zero-delay jump instruction. Moreover, when a conditional jump instruction is known in the PD stage, the IF unit extracts the offset field of the instruction, adds this value to the PC (program counter), and then fetches the branch target to the datapath chips. If compare is a fast compare (fcb), we can resolve the compare at the beginning of the ALU stage, that is, settle the branch before the IF stage of the next instruction. Therefore, we can obtain a zero-delay branch. In some cases, however, a full compare is necessary, so delayed or squashable compare and branch (dcb and scb) are addressed to reduce the penalty of pipeline drain. Experience has pointed out that only $10 \%$ of the slots are filled with no-operation instructions ${ }^{12}$. With the combination of fast and full compare and branch ( $\mathrm{fcb}$, $\mathrm{dcb}$, and scb) schemes, almost-zero-delay branch effect can be obtained.

\section{Non-delayed list access}

Most Lisp programs execute list access frequently ${ }^{20}$. List structure is usually constructed with two parts, the header of the list (car) and the tail of the list $(c d r)$. Each of the two parts contains a tag field to identify the data type and a data field. When a car or $c d r$ instruction is issued, tag field check and data field access are carried out simultaneously. Under LPU delayed RF mechanism, the register can be 
fetched via a short cut, and will incur no internal interlock to the following instruction, illustrated in Figure 10. The detailed timing in non-delayed load work is that the tag comparison stage $(\mathrm{cmp})$ is executed at the falling edge of phase 2 and the memory access stage (MA) of the previous instruction is also ready at this point, so that an internal forwarding from the MA stage to the $\mathrm{cmp}$ stage can be done.

\section{Non-delayed function call, jump and return}

Procedure calls occur frequently in Lisp programs. There are several problems associated with such frequent procedure calls. First, local variables should be kept with each function call and restored
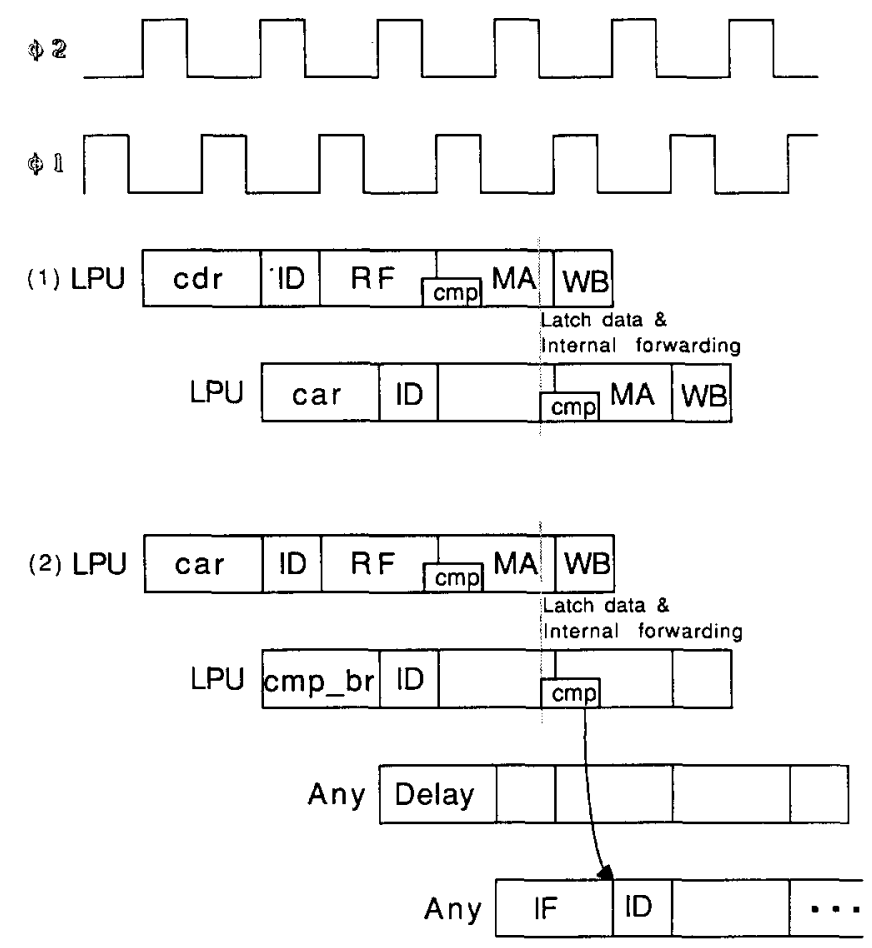

Figure 10 Non-delayed load execution from memory on every return. Second, arguments passing must be cross-referred to and from the memory. Frame windows are provided in the LPU to keep all these variables in registers to reduce the cost of external memory reference. The input frame in each procedure's frame window overlaps with the output frame of the procedure that calls it, and the output frame overlaps with the input frame of the procedure that it calls. Local variables are kept in the local frame of the procedure's window, and global ones in the global frame, which is visible as well as shared by the frame windows. This frame-window scheme makes procedure calls virtually free, and significantly speeds up their operation. However, the gain is not obtained without any price. Increasing the number of registers means that rather a large amount of chip area will be used, and register access time will be longer and process switching overheads will increase. Nevertheless, these prices are offset by a dedicated environment maintenance unit, that is, the LPU, and an independent execution pipeline stage designed between the IPU and the LPU.

\section{PERFORMANCE EVALUATION}

Six Gabriel benchmarks--a set of programs which test the speed of Lisp systems in various aspectshave been carried out to compare the performance of MARS with other architectures, as shown in Table 3. The first column shows the results for MARS, excluding the effect of cache misses. Columns 2-5 give the results for the other three architectures; the results for VAX-11/780 are from Gabriel's book ${ }^{21}$; SPUR's results are from Patterson's paper ${ }^{22}$; and MIPS-X's results are from Reference 23 , distributed into two columns-one without optimization, the other with optimization. The last four columns give the ratios of the execution

Table 3 Execution times in milliseconds for the Gabriel benchmarks (Note: MIPS-X@ mean that Lisp programs execute with optimization)

\begin{tabular}{|c|c|c|c|c|c|c|c|c|c|}
\hline & \multicolumn{5}{|c|}{ Time in milliseconds } & \multicolumn{4}{|c|}{ Ratios } \\
\hline & MARS & VAX & SPUR & MIPS-X & MIPS-X@ & $\begin{array}{l}\text { VAX/ } \\
\text { MARS }\end{array}$ & $\begin{array}{l}\text { SPUR/ } \\
\text { MARS }\end{array}$ & $\begin{array}{l}\text { MIPS-X/ } \\
\text { MARS }\end{array}$ & $\begin{array}{l}\text { MIPS-X@/ } \\
\text { MARS }\end{array}$ \\
\hline $\begin{array}{l}\text { tak } \\
\text { stak } \\
\text { takl } \\
\text { div-iter } \\
\text { div-rev } \\
\text { deriv }\end{array}$ & $\begin{array}{r}37 \\
70 \\
325 \\
55 \\
340 \\
110\end{array}$ & $\begin{array}{r}830 \\
7100 \\
5270 \\
3800 \\
3750 \\
8580\end{array}$ & $\begin{array}{r}120 \\
1060 \\
825 \\
- \\
2910 \\
990\end{array}$ & $\begin{array}{r}72 \\
602 \\
482 \\
307 \\
284 \\
604\end{array}$ & $\begin{array}{r}72 \\
592 \\
448 \\
157 \\
196 \\
381\end{array}$ & $\begin{array}{r}22.4 \\
101.4 \\
16.8 \\
69.1 \\
11.0 \\
78.0\end{array}$ & $\begin{array}{r}3.2 \\
15.1 \\
2.5 \\
- \\
8.6 \\
9.0\end{array}$ & $\begin{array}{l}1.9 \\
8.6 \\
1.5 \\
5.6 \\
0.8 \\
5.5\end{array}$ & $\begin{array}{l}1.9 \\
8.4 \\
1.4 \\
2.9 \\
0.6 \\
3.5\end{array}$ \\
\hline $\begin{array}{c}\text { Geometri } \\
\text { mean }\end{array}$ & & & & & & 35.4 & 6.2 & 2.9 & 2.2 \\
\hline
\end{tabular}


times of VAX-11/780, SPUR, and MIPS-X to the execution time of MARS. MARS executes Lisp programs about 35.4 times as fast as the VAX-11/780, almost 6.2 times as fast as SPUR, and about 2.2 times as fast as MIPS-X.

We found it interesting to trace the reasons for the performance difference between MARS and MIPS-X. Both MARS and MIPS-X are RISC processors, and of the same cycle time, but they differ in that MARS has a Lisp environment administrator (i.e. the LPU). The LPU has hardware support for tag handling, type checking on lists, binding registers, and uses frame windows to reduce the cost of register saving and restoring, and so forth. The MARS hardware for tag handling would eliminate about 25 percent of the cycles on MIPS-X, the binding register would also save about 50 percent of the cycles for load and store on MIPS-X, and the others (e.g. super-zero-jump, almost-zero-jump, fast list access, etc.) account for the remaining 45 percent. The frame windows do not function well for the Gabriel benchmarks and their average effect is small. The reasons are that some benchmarks use only a few arguments and local variables per frame window and have a call sequence directly backwards and forwards. Thus, the overhead of saving and restoring frame windows for overflows and underflows (which is 16 register-to-memory transfers instead of just several for MIPS-X) actually slows down some programs. Neither do the non-delayed $c a r$ and $c d r$ instructions in these benchmarks work well when compared with MIPS-X, since the delayed slot can always be filled. MARS will perform better on more realistic programs or cases without an optimization compiler involved, because the frame windows and nondelayed $c a r / c d r$ instructions will be more effective.

\section{CONCLUSION}

A design for a chip set for Lisp execution is proposed in this paper. By separating the IFU from the datapaths, and our deliberate pipeline arrangement, we can not only get co-ordinated execution among the IPU, FPU, and LPU but also drastically reduce slots due to control transfer; leaving the compiler more chance to fill the delayed load slots, thus accomplishing our goal of single-cycle instruction execution. What is more exciting, we can absorb the jump instructions within the IFU and directly issue the target to datapath chips to achieve what we call the super-zero-delay jump. An independent and separate LPU, playing the role of a Lisp runtime environment administrator, can accelerate Lisp programs for the following reasons. First, long complex register file access can be handled within the LPU, without a long accompanying ALU stage. Second, two independent pipeline executions of the IPU and LPU can separate the critical path of long register fetch plus integer processing into two different parts. Furthermore, the LPU can put off the register fetch until the external memory access is ready, and thus no delayed slot is needed when referring the data cache. Third, because instruction decoding of the IPU and LPU are local, some frequently used Lisp primitives can be hardwired without increasing the complexity and access time of instruction decode. Fourth, by excluding the arithmetic calculation within the LPU, the LPU can offer more silicon resource to accommodate more registers, and thus cut down the need for external memory access to increase system performance. Fifth, hardwired primitives can reduce the machine cycles needed when implemented by the underlying machine instruction.

\section{STATUS}

The implementation of the LPU is in progress. We have described the LPU at the register-transfer level, using the $\mathrm{M}$ modelling language. The layouts of the customized chips will be finished later in this year.

\section{REFERENCES}

1 Pleszkun, A. R. The architecture of Lisp machines. IEEE Computer, Mar. 1987, pp. 35-44

2 Moon, D. A. Architecture of the Symbolics 3600. Proc. 12th Symp. on Computer Architecture, Boston, June, 1985

3 Hill, M. et al. Design decisions in SPUR, IEEE Computer, Nov. 1986 , pp. 8-24

4 LMI. The Lambda System: Technical Summary, LISP Machines Inc., 1983

5 Davis, A. L. and Robison, S. V. The FAIM-1 symbolic multiprocessing system. Spring 1985 Compcon Digest of Papers, 1985 , pp. $370-375$

6 Yamaguchi, Y., Toda, K. and Yuba, T. A performance evaluation of a Lisp-based data-driven machine (EM-3). Proc. 10th Int. Symp. on Computer Architecture, June, 1983, pp. 363-369

7 Horowitz, M. et al. MIPS-X: A 20-MIPS peak, 32-bit, microprocessor with on-chip cache. IEEE J. Solid-State Circuits, SC-22 (5), 790-799 (Oct. 1987)

8 Chow, P. and Horowitz, M. Architectural tradeoffs in the design of MIPS-X. Proc. 13th Symp. on Computer Architecture, June, 1986, pp. $300-308$

9 Steenkiste, P. and Hennessy, J. Tags and type checking in LISP hardware and software approaches. Proc. 2nd Int. Conf. on Architecture Support for Programming Languages and Operating Systems, ACM/IEEE, October, 1987, pp. 50-59

10 Jang, Gia-Shuh, Lai, Feipei, Lee, Hung-Chang, Maa, Y. C., Parng, T. M. and Tsai, Jenn-Huan. MARS Multiprocessor Architecture Reconciling Symbolic with numerical processing. Int. Symp. on VLSI Technology, Systems, and Applications, May 17-19, 1989 
11 Katevenis, M. Reduced instruction set computer architectures for VLSI. Ph.D. Dissertation, Computer Science Division (EECS), UCB/CSD, University of California, Berkeley, Oct. 1983

12 Lee, Hung-Chang and $\mathrm{Wu}$, We-Hung. Lock-up free cache design and the phoenix protocol. Memo \#329-1, Computer Science Division (EECS), National Taiwan University, Taiwan, R.O.C., Jan. 1989

13 Tsai, Jenn-Yuan. The design of list processing unit ( $L P U$ ) for the MARS system. M.Sc. Thesis, Computer Science Division (EECS), National Taiwan University, Taiwan, R.O.C., July 1989

14 McFarling, S. and Hennessy, J. Reducing the cost of branches. Proc. 13th Symp. on Computer Architecture, June, 1986 pp. 396-403

15 Smith, J. E. A study of branch prediction strategies, Proc. 8th Symp. on Computer Architecture, May, 1981, pp. 135-148

16 Lee, J. K. F. and Smith, A. J. Branch prediction strategies and branch target buffer design. IEEE Computer, Jan. 1984, pp. 6-22

17 Hennessy, J. et al. Hardware/software tradeoffs for increased performance, Proc. SIGARCH/SIGPLAN Symp. on Architectural Support for Programming Language and Operating Systems, ACM, Palo Alto, March, 1982, pp. 2-11

18 Lilja, D. J. Reducing the branch penalty in pipelined processors IEEE Computer, Jul. 1988, pp. 47-55

19 Ditzel, D. and McLellan, H. R. Branching flooding in the CRISP microprocessor: Reducing branch delay to zero. In Proc. 14th Annual Symp. on Computer Architecture, June, 1987, pp. 2-9

20 Clark, D. W. Measurements of dynamic list structure use in Lisp. IEEE Trans. Software Engineering, SE-5 (1), (Jan. 1979)

21 Gabriel, R. P. Performance and Evaluation of Lisp System. The MIT Press, Cambridge, MA (1985)

22 Taylor, G. S., Patterson, D. A. et al. Evaluation of the SPUR Lisp architecture. Proc. 13th Int. Symp. on Computer Architecture, June, 1986 , pp. $444-452$.

23 Steenkiste, P. and Hennessy, J. Lisp on a reduced instruction set processor: Characterization and optimization. IEEE Computer, June 1988 , pp. 34-45 